\title{
ESTUDO DA PRESENÇA DE VANÁDIO E MOLIBDÊNIO SOBRE NIÓBIA NA DESIDROGENAÇÃO OXIDATIVA DO PROPANO
}

\author{
B. D. $\operatorname{COSTA}^{1}$, J.A.J. RODRIGUES ${ }^{2}$ e G. G. CORTEZ $^{1}$ \\ ${ }^{1}$ Escola de Engenharia de Lorena - USP - Departamento de Engenharia Química \\ ${ }^{2}$ Laboratório de Combustão e Propulsão - Instituto Nacional de Pesquisas Espaciais \\ E-mail para contato: cortez@ dequi.eel.usp.br
}

\begin{abstract}
RESUMO - A desidrogenação oxidativa do propano (DOP) apresenta significante vantagem energética em comparação com o atual processo de produção de olefinas, o desafio comercial está em atingir elevados rendimentos, já que o propeno formado é oxidado para óxidos de carbono mais estáveis $\left(\mathrm{CO}\right.$ e $\left.\mathrm{CO}_{2}\right)$. O objetivo de estudo deste trabalho, foi avaliar a atividade catalítica do sistema $x \mathrm{Mo}-y \mathrm{~V} / \mathrm{Nb}_{2} \mathrm{O}_{5}$ na DOP. $\mathrm{O} \mathrm{Nb}_{2} \mathrm{O}_{5}$ foi preparado pelo método hidrotérmico e a impregnação dos óxidos de molibdênio e vanádio foram por impregnação úmida com excesso de solvente, formando três catalisadores, que foram caracterizados pelas técnicas de volumetria de $\mathrm{N}_{2}$, RTP, DRX e pela reação de desidrogenação oxidativa de propano. A adição gradual de vanádio e de molibdênio gerou uma diminuição no volume de poros e na área superficial específica do catalisador. Através da reação de DOP observou-se um aumento na conversão de propano e rendimento de propeno e uma diminuição no rendimento de $\mathrm{CO}_{2}$ a partir da adição de $\mathrm{V}$ e Mo ao $\mathrm{Nb}_{2} \mathrm{O}_{5}$.
\end{abstract}

PALAVRAS-CHAVE: Vanádio; Molibdênio; Nióbio; DOP; Propano.

\section{INTRODUÇÃO}

A desidrogenação oxidativa de alcanos é uma rota alternativa para a produção de alcenos a partir de alcanos devido ao grande consumo energético, encontrados na desidrogenação catalítica e no craqueamento a vapor, para a produção de olefinas (Mamedov e Cortes-Corberan, 1995). Os esforços atuais estão concentrados na concepção de catalisadores mais eficientes, os quais produzem propeno com rendimentos mais elevados em maiores conversões.

$\mathrm{O}$ emprego de $\mathrm{Nb}_{2} \mathrm{O}_{5}$ como suporte em catalisadores que contenham vanádio pode representar uma alternativa sinergética, uma vez que os dois elementos além de pertencerem ao mesmo grupo da tabela periódica, apresentam várias propriedades químicas semelhantes (Chary et. al. 1999). Óxidos metálicos do grupo $\mathrm{V}$ suportados têm uma grande variedade de aplicações catalíticas e têm sido extensivamente investigados nos últimos anos (Cortez et. al., 2003). Catalisadores de $\mathrm{V}_{2} \mathrm{O}_{5}-\mathrm{MoO}_{3}$ tem sido largamente usados em várias reações de oxidação seletiva (Satsuma et. al., 1991)

Neste contexto, o presente trabalho tem como objetivo principal avaliar as características texturais de catalisadores de molibdênio e vanádio suportados em nióbia, preparada a partir do 


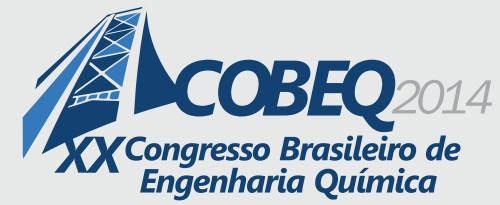

método hidrotérmico, e a atividade catalítica na desidrogenação oxidativa de propano.

\section{EXPERIMENTAL}

A caracterização do $\mathrm{Nb}_{2} \mathrm{O}_{5}$ e dos catalisadores foi realizada através das técnicas de volumetria de nitrogênio, difratometria de raios $\mathrm{X}$ e redução à temperatura programada. A atividade catalítica foi analisada através da reação de desidrogenação oxidativa do propano.

\subsection{SÍNTESE DOS CATALISADORES}

Neste trabalho, foram sintetizados três catalisadores, identificados como $1 \mathrm{Mo}-7 \mathrm{~V} / \mathrm{Nb}, 2 \mathrm{Mo}-$ $6 \mathrm{~V} / \mathrm{Nb}$ e $3 \mathrm{Mo}-5 \mathrm{~V} / \mathrm{Nb}$. O óxido de nióbio $\left(\mathrm{Nb}_{2} \mathrm{O}_{5}\right)$, ou nióbia, foi preparado a partir do ácido nióbico HY 340, o qual foi fornecido pela Companhia Brasileira de Metalurgia e Mineração (CBMM). Para a síntese do suporte, o ácido nióbico foi submetido a um tratamento hidrotérmico.

A impregnação do molibdênio e do vanádio no $\mathrm{Nb}_{2} \mathrm{O}_{5}$ foi realizada por co-impregnação úmida com excesso de solvente (água). Na preparação dos catalisadores $x \mathrm{Mo}-y \mathrm{~V} / \mathrm{Nb}_{2} \mathrm{O}_{5}$, utilizou-se uma solução aquosa contendo $\mathrm{NH}_{4} \mathrm{VO}_{3} \mathrm{e}\left(\mathrm{NH}_{4}\right)_{6} \mathrm{Mo}_{7} \mathrm{O}_{24} \cdot 4 \mathrm{H}_{2} \mathrm{O}$. As massas dos sais foram calculadas de forma que a quantidade de molibdênio e de vanádio por área do suporte seja igual a um total de 8 átomos, sendo $x=1,2$ e 3 átomos de $\mathrm{Mo} / \mathrm{nm}^{2} \mathrm{e} \mathrm{y}=5,6$ e 7 átomos de $\mathrm{V} / \mathrm{nm}^{2}$ sobre $\mathrm{o} \mathrm{Nb}_{2} \mathrm{O}_{5}$ calcinado a $500^{\circ} \mathrm{C}$. Após a dissolução dos sais no balão do rota-evaporador, foi adicionada a massa do óxido de nióbio previamente calculada para a impregnação. Após um período de uma hora, o solvente foi retirado a $70^{\circ} \mathrm{C}$ sob vácuo. A etapa seguinte à impregnação foi a calcinação, na qual os catalisadores foram secos a $110^{\circ} \mathrm{C}$ por 24 horas em estufa e, depois, calcinados a $500^{\circ} \mathrm{C}$ por 4 horas em mufla.

\subsection{CARACTERIZAÇÃO FISICO-QUÍMICA}

As análises por difratometria de raios X (DRX) foram realizadas empregando-se o método do pó, utilizando-se um equipamento da marca Panalytical, modelo Enpyrean, radiação CuK $\alpha$ obtida em $40 \mathrm{kV}$. Os difratogramas de raios $\mathrm{X}$ foram obtidos com ângulo de varredura de $2 \theta$ variando de 5 a $90^{\circ}$. As fases cristalinas foram identificadas com auxílio da biblioteca do JCPDS - Joint Committee of Powder Diffraction Standards (PCPDFWIN, 2002).

As medidas de áreas superficiais específicas, volume de poros (método BET) e distribuição de volume de poros (método $\mathrm{BJH}$ ) foram determinadas a partir das isotermas de adsorção-dessorção de $\mathrm{N}_{2}$ a $-196^{\circ} \mathrm{C}$, utilizando-se um equipamento da marca BEL Japan, INC., modelo BelSorp mini II. As amostras foram previamente tratadas a $300^{\circ} \mathrm{C}$, por $1 \mathrm{~h}$, em nitrogênio.

O perfil de redução das espécies redutíveis presentes nos catalisadores foi determinado pela técnica de redução à temperatura programada (RTP), utilizando-se um equipamento da marca Quantachrome, modelo Chembet-3000, equipado com um DCT. As amostras (15 mg) foram secas in situ a $200^{\circ} \mathrm{C}$ por $1 \mathrm{~h}$ sob fluxo de hélio e em seguida resfriados à temperatura ambiente e, em seguida, reduzidos até $750^{\circ} \mathrm{C}\left(10^{\circ} \mathrm{Cmin}^{-1}\right)$ com uma mistura de $10 \% \mathrm{H}_{2} / \mathrm{N}_{2}\left(30 \mathrm{mLmin}^{-1}\right)$. 


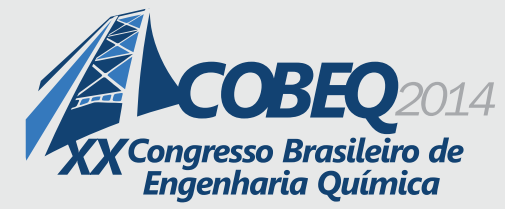

Os catalisadores $(100 \mathrm{mg}$ ) foram avaliados na reação de desidrogenação oxidativa do propano entre as temperaturas de reação de 300 a $520^{\circ} \mathrm{C}$. Foram adicionados $200 \mathrm{mg}$ de óxido misto de Mo$\mathrm{Nb}$ (inerte à DOP) e misturados ao catalisador no reator com o objetivo de melhorar a difusão das moléculas de propano sobre a superfície do catalisador. A mistura de gases alimentada ao reator foi de $\mathrm{O}_{2} / \mathrm{C}_{3} \mathrm{H}_{8} / \mathrm{He}(3 / 2 / 6$ molar) com um fluxo total de $45 \mathrm{~mL} / \mathrm{min}$. O propano e os produtos não convertidos foram analisados por um cromatógrafo gasoso em linha, modelo Varian 450, equipado com um TCD, utilizando-se uma coluna Cromossorb 107 e uma peneira molecular 4A. Os produtos principais de reação foram propeno, água, e $\mathrm{CO}_{2}$. A conversão do propano foi calculada a partir de um balanço de quantidade de carbono, assumindo como sendo constante o balanço de carbono presente na saída do reator e no fluxo de alimentação do reagente. Baseado nas concentrações de entrada e saída e assumindo as condições de um reator diferencial, a conversão total $\left(\mathrm{X}_{\mathrm{A}}\right)$, a seletividade $\left(S_{p}\right)$ e o rendimento aos produtos $\left(R_{p}\right)$ foram calculados através das seguintes equações:

$$
\mathrm{X}_{\mathrm{A}}(\%)=\left(\frac{\mathrm{n}_{\mathrm{R}}}{\mathrm{n}_{\mathrm{R} 0}}\right) \cdot 100 \quad \mathrm{~S}_{\mathrm{p}}(\%)=\left(\frac{\mathrm{n}_{\mathrm{P}}}{\mathrm{n}_{\mathrm{I}}}\right)\left(\frac{\mathrm{N}_{\mathrm{CP}}}{\mathrm{N}_{\mathrm{CR}}}\right) \cdot 100 \quad \mathrm{R}_{\mathrm{p}}(\%)=\mathrm{X}_{\mathrm{A}} \cdot \mathrm{S}_{\mathrm{p}}
$$

onde $\mathrm{n}_{\mathrm{R}}, \mathrm{n}_{\mathrm{R} 0}$ são os números de moles do reagente consumidos e alimentados, respectivamente; $\mathrm{n}_{\mathrm{P}}$ o número de moles dos produtos formados; e $\mathrm{N}_{\mathrm{CP}}$ e $\mathrm{N}_{\mathrm{CR}}$ são os números de átomos de carbono presentes nos produtos formados e no reagente alimentado respectivamente.

\section{RESULTADOS E DISCUSSÃO}

As áreas superficiais específicas $(\mathrm{Sg})$, volume total de poros $(\mathrm{Vp})$ e diâmetro médio dos poros (Dp) do suporte e dos catalisadores $x \mathrm{Mo}-y \mathrm{~V} / \mathrm{Nb}$ são apresentados na Tabela 1.

Tabela 1 - Propriedades texturais das amostras calcinadas.

\begin{tabular}{cccccc}
\hline Amostras & $\% \mathrm{Mo}(\mathrm{p} / \mathrm{p})$ & $\% \mathrm{~V}(\mathrm{p} / \mathrm{p})$ & $\mathrm{Sg}\left(\mathrm{m}^{2} / \mathrm{g}\right)$ & $\mathrm{Vp}\left(\mathrm{cm}^{3} / \mathrm{g}\right)$ & $\mathrm{Dp}(\mathrm{nm})$ \\
\hline $\mathrm{Nb}_{2} \mathrm{O}_{5}$ & 0 & 0 & 74,0 & 0,19 & 10,0 \\
$1 \mathrm{Mo}-7 \mathrm{~V} / \mathrm{Nb}$ & 1,14 & 3,85 & 17,0 & 0,10 & 25,0 \\
$2 \mathrm{Mo}-6 \mathrm{~V} / \mathrm{Nb}$ & 2,08 & 3,31 & 9,0 & 0,06 & 27,0 \\
$3 \mathrm{Mo}-5 \mathrm{~V} / \mathrm{Nb}$ & 3,11 & 3,11 & 6,0 & 0,05 & 29,0 \\
\hline
\end{tabular}

$\mathrm{A}$ adição de $\mathrm{Mo}$ e de $\mathrm{V}$ ao $\mathrm{Nb}_{2} \mathrm{O}_{5}$ proporcionou diminuição nos valores da área superficial específica e do volume de poros, efeito este provocado pela possível obstrução dos poros de menor diâmetro, mas observou-se também que adição crescente de molibdênio influencia diretamente neste efeito, já que o Mo possui raio atômico maior que o V. Além disso, a presença do Mo e $\mathrm{V}$ no interior dos poros do suporte causa um colapso durante o tratamento térmico, resultando em um aumento nos valores dos diâmetros médios dos poros no suporte. 


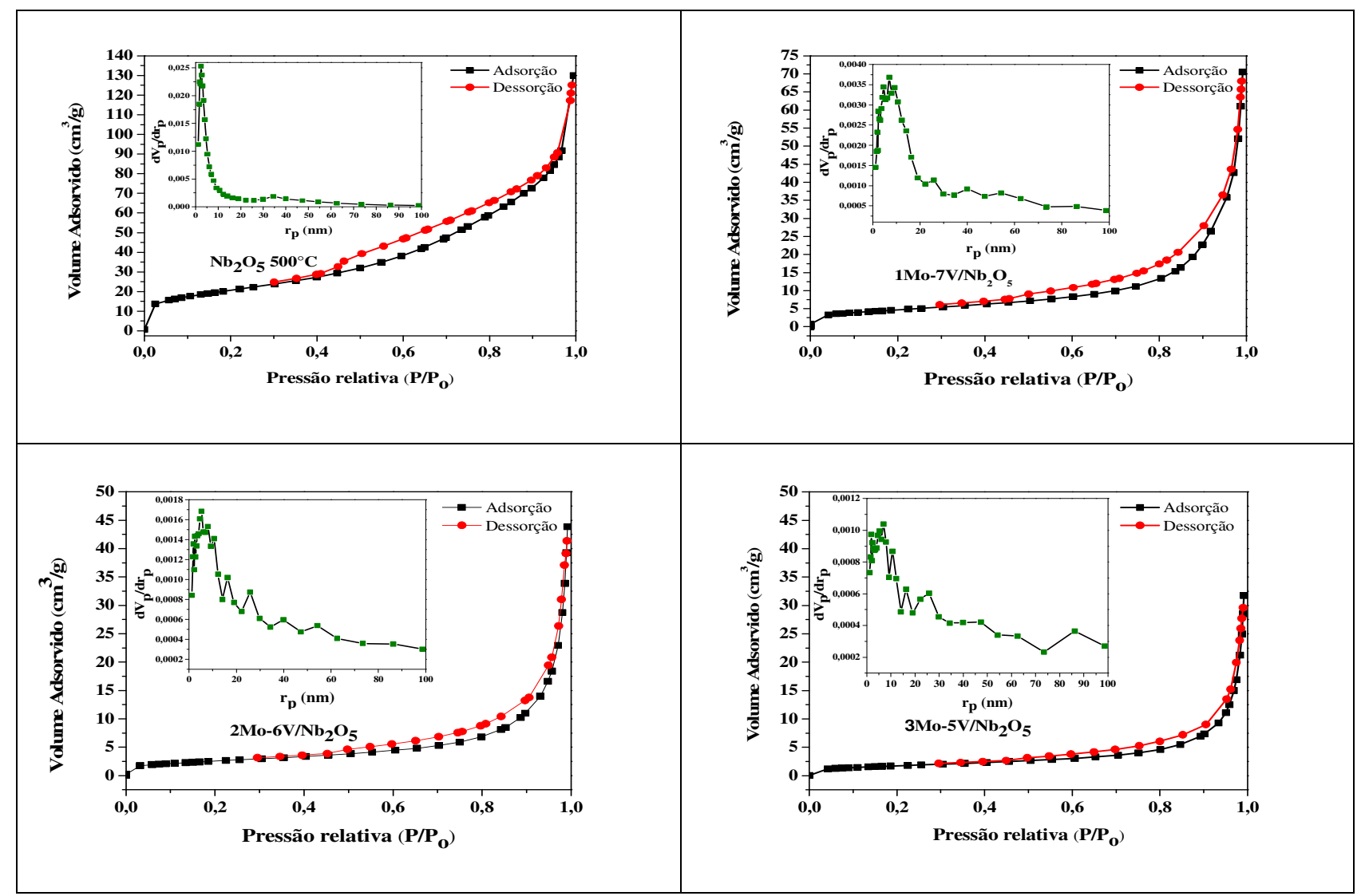

Figura 1 - Isotermas de adsorção-dessorção de $\mathrm{N}_{2}$ e distribuição de volume de poros do suporte e dos catalisadores $x \mathrm{Mo}-y \mathrm{~V} / \mathrm{Nb}$.

As isotermas de adsorção-dessorção de $\mathrm{N}_{2}$ a $-196^{\circ} \mathrm{C}$ e as curvas de distribuição de poros do suporte e dos catalisadores são apresentadas na Figura 1. Observa-se a predominância de isotermas do tipo IV, que segundo a nomenclatura da IUPAC, são típicas de materiais mesoporosos (Roqueirol et $a l ., 1994)$. Nota-se também, que a adição de molibdênio e de vanádio sobre o suporte não modificou o perfil das isotermas. Observa-se um "laço" de histerese do tipo $\mathrm{H} 3$ a $0,40<\mathrm{P} / \mathrm{P}_{0}<0,9$ nas isotermas entre as etapas de adsorção-dessorção associado à condensação capilar em poros na forma de fendas.

$\mathrm{O}$ DRX do $\mathrm{Nb}_{2} \mathrm{O}_{5}$ e dos catalisadores são apresentados pelas Figuras 2 e 3, respectivamente. A nióbia calcinada a $500^{\circ} \mathrm{C}$ apresenta estrutura amorfa, tendo início um processo de formação de planos cristalográficos ordenados no pico mais intenso em $2 \theta=22,69^{\circ}$, característica da fase TT ou T do $\mathrm{Nb}_{2} \mathrm{O}_{5}$, resultado obtido também em estudos de Chary et. al. (2003). A adição de $\mathrm{MoO}_{3}$ e $\mathrm{V}_{2} \mathrm{O}_{5}$ não provoca mudanças no perfil do DRX, o que indica que os óxidos estão presentes em um estado amorfo, ou altamente dispersos sobre a nióbia, porém não se descarta a possibilidade de haver cristalitos desses óxidos com dimensões inferiores a $4 \mathrm{~nm}$, uma vez que esse é o limite inferior de detecção de estruturas cristalinas pela técnica de DRX (Chary et. al., 2003). 


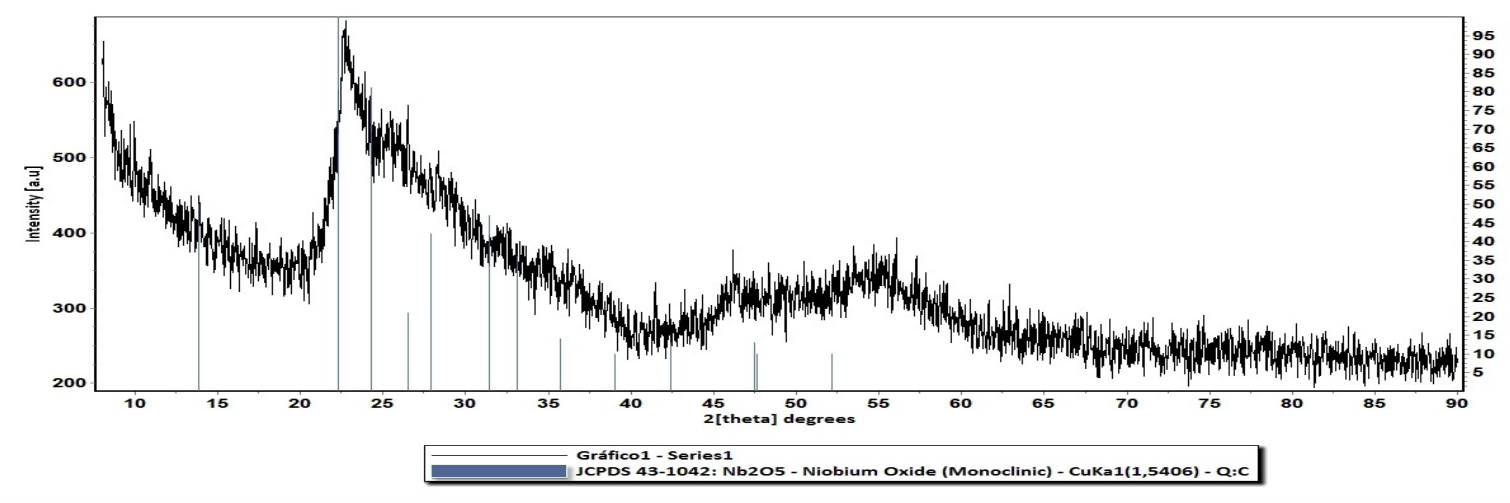

Figura 2 - Difratometria de Raios- $\mathrm{X}$ do $\mathrm{Nb}_{2} \mathrm{O}_{5}$ calcinado à $500^{\circ} \mathrm{C}$

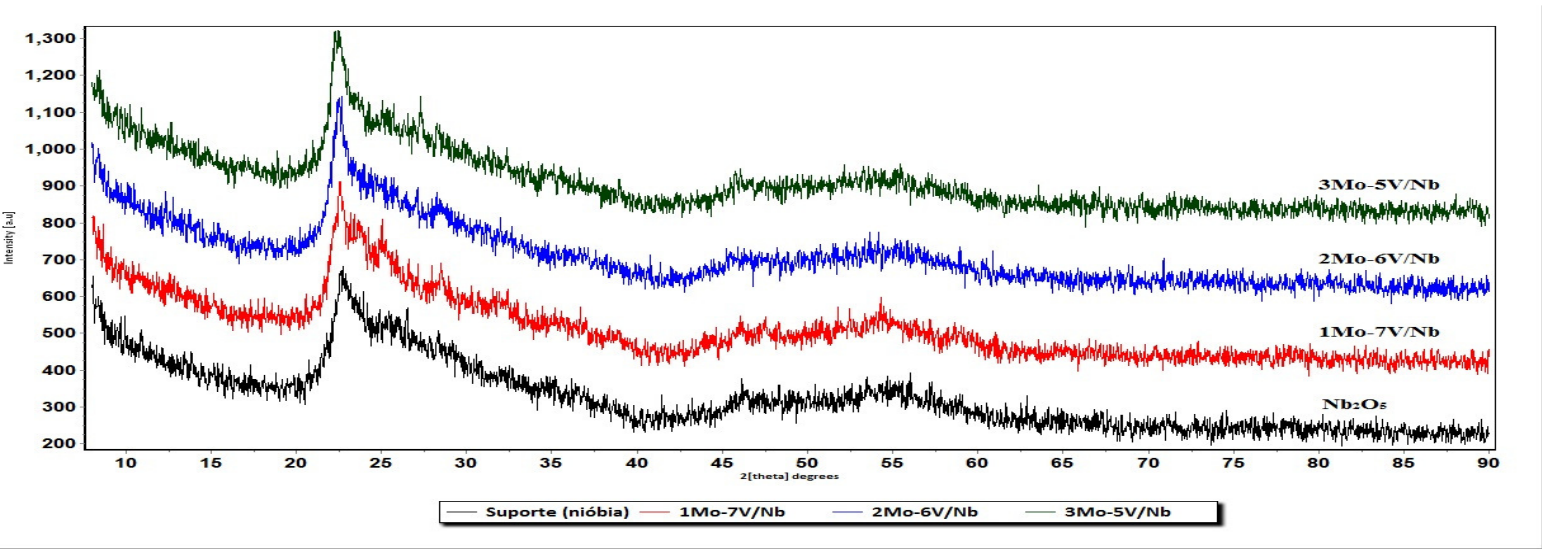

Figura 3 - Difratometria de Raios-X dos catalisadores e $\mathrm{Nb}_{2} \mathrm{O}_{5}$ calcinados à $500^{\circ} \mathrm{C}$

As análises de redução à temperatura programada (RTP) dos catalisadores são apresentadas na Figura 4. Os catalisadores $1 \mathrm{Mo}-7 \mathrm{~V} / \mathrm{Nb}, 2 \mathrm{Mo}-6 \mathrm{~V} / \mathrm{Nb}$ e $3 \mathrm{Mo}-5 \mathrm{~V} / \mathrm{Nb}$ apresentaram um pico de redução em torno de $660^{\circ} \mathrm{C}$. A intensidade do pico aumenta conforme aumenta a quantidade de molibdênio e diminui a de vanádio na estrutura. A presença de apenas um pico mais intenso de redução pode indicar uma interação entre o molibdênio e o vanádio e destes com o nióbio, formando um óxido misto, já que o nióbio mássico reduz a temperaturas mais elevadas $\left(>800^{\circ} \mathrm{C}\right)$. A redução do vanádio mássico ocorre acima de $650^{\circ} \mathrm{C}$ com um pico bem definido e a redução do molibdênio mássico ocorre acima de $750^{\circ} \mathrm{C}$. $\mathrm{O} \mathrm{V}_{2} \mathrm{O}_{5}$ mássico apresenta três temperaturas máximas de redução a 655 , 690 e $823^{\circ} \mathrm{C}$, que corresponde a seguinte etapa de redução das espécies de vanádio, descrito por Koranne et. al. (1994): $\mathrm{V}_{2} \mathrm{O}_{5} \rightarrow \mathrm{V}_{6} \mathrm{O}_{13}\left(675^{\circ} \mathrm{C}\right) ; \mathrm{V}_{6} \mathrm{O}_{13} \rightarrow \mathrm{V}_{2} \mathrm{O}_{4}\left(705^{\circ} \mathrm{C}\right) ; \mathrm{V}_{2} \mathrm{O}_{4} \rightarrow \mathrm{V}_{2} \mathrm{O}_{3}\left(780^{\circ} \mathrm{C}\right)$. De acordo com Arnoldy et.al. (1985), a redução do molibdênio ocorre essencialmente em duas etapas, cujos picos foram atribuídos à seguinte sequência de redução: $\mathrm{MoO}_{3} \rightarrow \mathrm{MoO}_{2}\left(765^{\circ} \mathrm{C}\right) ; \mathrm{MoO}_{2} \rightarrow \mathrm{Mo}\left(997^{\circ} \mathrm{C}\right)$. $\mathrm{Na}$ primeira etapa, a temperatura de $765^{\circ} \mathrm{C}$ corresponde à redução da espécie $\mathrm{Mo}^{+6}$ à espécie $\mathrm{Mo}^{+4}, \mathrm{e}$ na segunda o molibdênio é reduzido para o estado metálico (Mo) em temperatura de $997^{\circ} \mathrm{C}$. 


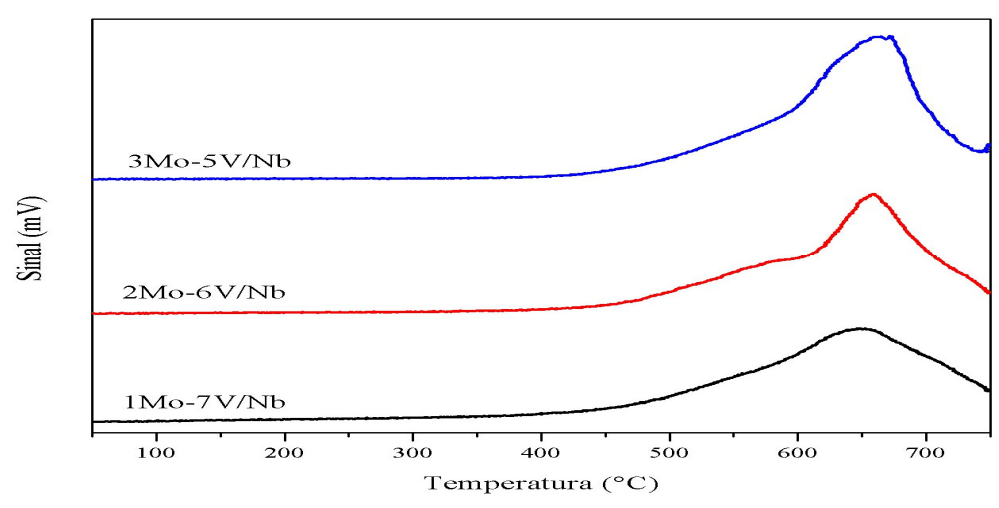

Figura 4 - Perfil de RTP dos catalisadores em $10 \% \mathrm{H}_{2} / \mathrm{N}_{2}$

A Tabela 2 apresenta os valores de conversão do propano $\left(\mathrm{X}_{\mathrm{A}}\right)$, rendimento à propeno $\left(\mathrm{R}_{\mathrm{p}}\right)$ e seletividade à propeno $\left(\mathrm{S}_{\mathrm{p}}\right)$ para três temperaturas dos catalisadores $1 \mathrm{Mo}-7 \mathrm{~V} / \mathrm{Nb}, 2 \mathrm{Mo}-6 \mathrm{~V} / \mathrm{Nb}$ e $3 \mathrm{Mo}-$ $5 \mathrm{~V} / \mathrm{Nb}$ e do $\mathrm{Nb}_{2} \mathrm{O}_{5}$ na reação de desidrogenação oxidativa do propano (DOP). Em relação ao $\mathrm{Nb}_{2} \mathrm{O}_{5}$, a adição de molibdênio e de vanádio proporcionou um aumento na atividade catalítica, porém a seletividade a $\mathrm{CO}_{2}$ também aumentou. $\mathrm{O} \mathrm{Nb}_{2} \mathrm{O}_{5}$ apresentou seletividade ao propeno até $510^{\circ} \mathrm{C}$, temperatura na qual se verificou a maior quantidade de $\mathrm{CO}_{2}$ formado, indicando um alto grau de combustão no processo. Chen et al. (1999) analisaram a composição isotópica dos produtos, através da análise de Carbono-13 $\left({ }^{13} \mathrm{C}\right)$, durante a reação de desidrogenação oxidativa do propano, utilizandose $\mathrm{VO}_{\mathrm{x}} / \mathrm{ZrO}_{2}$ como catalisador. Os autores verificaram que a fração de ${ }^{13} \mathrm{C}$ no $\mathrm{CO}$ foi similar à encontrada no propeno, em todos os tempos de reação, enquanto que o conteúdo isotópico no $\mathrm{CO}_{2}$ foi ligeiramente maior do que o observado no $\mathrm{CO}$ e no propeno. Portanto, eles concluíram que o $\mathrm{CO}$ é produzido somente pela oxidação secundária do propeno e o $\mathrm{CO}_{2}$ é formado tanto pela oxidação secundária da olefina, quanto pela combustão direta do propano.

Entre os catalisadores, o $2 \mathrm{Mo}-6 \mathrm{~V} / \mathrm{Nb}$ apresentou uma maior conversão e rendimento e seletividade à propeno. $\mathrm{O}$ vanádio possui múltiplos estados de oxidação, o que facilita a conversão para óxidos de diferentes estequiometrias, devido aos processos de oxidação e redução. Acredita-se que esse seja o fator fundamental para sua atividade e seletividade. Pentóxido de vanádio mássico possui ainda características superficiais redox $(>90 \%)$, enquanto que $\mathrm{Nb}_{2} \mathrm{O}_{5}$ mássico possui exclusivamente características superficiais ácidas (>100\%) (Ko e Weissman, 1990). No estudo de TPD de $\mathrm{NH}_{3}$ adsorvida sobre $\mathrm{Mo} / \gamma-\mathrm{Al}_{2} \mathrm{O}_{3}$, descrito por Abello et. al., (2001), observou-se que a interação com $\mathrm{NH}_{3}$ aumenta após a adição de Mo sobre $\gamma-\mathrm{Al}_{2} \mathrm{O}_{3}$ e a força dos sítios ácidos muda. Nos resultados observados, a presença de vanádio e molibdênio pode ter gerado novos sítios com forças ácidas fracas e moderadas sobre a nióbia. A relação entre a concentração de reagentes e produtos na reação de ODH de propano leva a crer que esta reação ocorra via um mecanismo redox do tipo Marsvan Krevelen, no qual a ativação irreversível da ligação C-H ocorre na superfície do catalisador, próximo a átomos de oxigênio reticulares e a grupos hidroxila terminais (Doornkamp e Ponec, 2000). Dentre os catalisadores estudados, a reação DOP envolve ciclos redox e etapas de ativação da ligação $\mathrm{C}-\mathrm{H}$, que requer transferência de elétrons do oxigênio ao vanádio em domínios de $\mathrm{VO}_{\mathrm{x}}$ superficiais. Portanto, a presença de vanádio faz, portanto, com que o produto desejado, o propeno, seja fortemente 
adsorvido e oxidado à $\mathrm{CO}_{2}$ a altas temperaturas de reação (Mamedov e Cortes-Corberan, 1995).

Tabela 2 - Resultados da atividade catalítica na ODH do propano.

\begin{tabular}{cccccc}
\hline Amostras & $\mathrm{T}_{\text {Reação }}\left({ }^{\circ} \mathrm{C}\right)$ & $\mathrm{X}_{\mathrm{A}}(\%) \mathrm{C}_{3} \mathrm{H}_{8}$ & $\mathrm{R}_{\mathrm{p}}(\%) \mathrm{C}_{3} \mathrm{H}_{6}$ & $\mathrm{~S}_{\mathrm{p}}(\%) \mathrm{C}_{3} \mathrm{H}_{6}$ & $\mathrm{~S}_{\mathrm{p}}(\%) \mathrm{CO}_{2}$ \\
\hline \multirow{2}{*}{$\mathrm{Nb}_{2} \mathrm{O}_{5}$} & 486 & 10,7 & 9,8 & 91,7 & 3,5 \\
& 498 & 14,7 & 13,3 & 90,5 & 2,7 \\
& 510 & 24,1 & 20,9 & 86,7 & 20,0 \\
\hline \multirow{3}{*}{$1 \mathrm{Mo}-7 \mathrm{~V} / \mathrm{Nb}$} & 482 & 20,7 & 16,7 & 81,0 & 19,0 \\
& 492 & 26,5 & 22,1 & 83,5 & 16,5 \\
& 504 & 38,6 & 33,0 & 85,3 & 14,7 \\
\hline \multirow{2}{*}{$2 \mathrm{Mo}-6 \mathrm{~V} / \mathrm{Nb}$} & 480 & 22,4 & 20,7 & 92,6 & 7,4 \\
& 493 & 36,1 & 32,3 & 89,5 & 10,5 \\
& 504 & 61,9 & 51,7 & 83,5 & 16,5 \\
\hline \multirow{3}{*}{$3 \mathrm{Mo}-5 \mathrm{~V} / \mathrm{Nb}$} & 480 & 15,7 & 13,8 & 88,1 & 7,0 \\
& 493 & 24,9 & 21,0 & 84,3 & 6,6 \\
& 506 & 44,7 & 33,9 & 75,9 & 7,2 \\
\hline
\end{tabular}

\section{CONCLUSÕES}

O óxido de nióbio se mostrou favorável para ser utilizado como catalisador e suporte, devido a sua alta temperatura de redução, tornando-o mais estável, e promovendo assim uma forte interação com o vanádio e molibdênio. A propriedade textural da nióbia foi modificada pela adição de molibdênio e de vanádio, a qual provocou o bloqueio dos microporos pelas espécies superficiais. A metodologia empregada na preparação do $\mathrm{Nb}_{2} \mathrm{O}_{5}$ revelou um material amorfo ao DRX e a adição de $\mathrm{MoO}_{3}$ e $\mathrm{V}_{2} \mathrm{O}_{5}$ não provocou mudanças no perfil do DRX, o que indica que os óxidos estão presentes em um estado amorfo, ou altamente dispersos sobre o suporte. Os resultados de TPR revelaram que a quantidade de Mo e de V não alterou os perfis de redução significativamente e há presença de picos mais intensos de redução, mas que não corresponde aos picos do vanádio mássico ou do molibdênio mássico, o que pode indicar uma interação entre o molibdênio e o vanádio e estes com o nióbio, formando óxidos mistos. Nos resultados da reação de DOP, verificou-se que a presença de Mo e V sobre $\mathrm{Nb}_{2} \mathrm{O}_{5}$ proporcionou aumento na conversão do propano, sendo mais seletivo o catalisador com quantidades intermediárias de Mo e V (2Mo-6V/Nb).

\section{AGRADECIMENTOS}

Ao Laboratório de Combustão e Propulsão (LCP) do Instituto Nacional de Pesquisas Espaciais (INPE/Cachoeira Paulista) pelo uso de sua infra-estrutura na síntese e caracterização da nióbia e a FAPESP pela bolsa de Iniciação Científica concedida a Bianca Daniela Costa (Proc. 2012/23064-1). 


\section{REFERÊNCIAS}

ABELLO, M.C.; GOMEZ, M.F.; FERRETTI, O. Mo/ $\gamma-\mathrm{Al}_{2} \mathrm{O}_{3}$ catalysts for the oxidative dehydrogenation of propane.: Effect of Mo loading. Appl. Catal. A General,v. 207, p. 421-431, 2001.

ARNOLDY, P.; JONGE, J. C. M.; MOULJIN, J. A. Temperature-Programmed Reduction of $\mathrm{MoO}_{3}$ and $\mathrm{MoO}_{2}$. J. Phys. Chem., v. 89, p. 4517-4526, 1985.

CHARY, K.V.R.; KISHAN, G.; BHASKAR, T. Dispersion and reactivity of vanadium oxide catalysts supported on niobia. Chem. Comm. v. 15, p.1399-1400, 1999.

CHARY, K.V.R.; RAMESH, K.; VIDYASAGAR, G.; RAO, V. V. Vapour phase alkylation of phenol with methanol over vanadium oxide supported on zirconia. J. Mol. Catal. A: Chem., v.198, p. 195-204, 2003.

CHEN, K. D.; KHODAKOV, A.; YANG, J.; BELL, A. T.; IGLESIA, E. Isotopic tracer and kinetic studies of oxidative dehydrogenation pathways on vanadium oxide catalysts. J. Catal., v. 186, n. 2, p. 325-333, 1999.

CORTEZ, G.G.; FIERRO, J.L.G.; BAÑARES, M.A. Role of potassium on the structure and activity of alumina-supported vanadium oxide catalysts for propane oxidative dehydrogenation Catal. Today, v. 78, p.219-228, 2003.

DOORNKAMP, C.; PONEC, V. The universal character of the Mars and Van Krevelen mechanism. J. Mol. Catal. A: Chem., v. 162, n. 1-2, p. 19-32, 2000.

KO, E.I.; WEISSMAN, J. G. Structures of niobium pentoxide and their implications on chemical behavior. Catal. Today, v.8, p. 27-36, 1990

KORANNE, M.M.; GOODWIN, J. G.; MARCELIN, G. Characterization of silica- and aluminasupported vanadia catalysts using temperature programmed reduction. J. Catal., v. 148, p. 369-377, 1994.

MAMEDOV, E.A.; CORTES-CORBERAN, V. Oxidative Dehydrogenation of lower alkanes on vanadium oxide-based catalysts. The present state of the art and outlooks. Appl. Catal. A., v.127, p. 140, 1995.

PCPDFWIN v. 2.3; Program for Powder Diffraction Files, JCPDS, International Center for Diffraction Data, Pensylvania, USA, 2002 (CD-ROM).

ROQUEIROL, J.; RODRIGUEZ-REINOSO, F.; SING, S.W. in: Characterization of porous solids III, Amsterdam: Elsevier, 1994.

SATSUMA, A.; OKADA, F.; HATTORI, A.; MIYAMOTO, A.; HATTORI, T.; MURAKAMI, Y. Promotion effects of various oxides on oxidation of benzene over vanadium pentoxide catalysts. Appl. Catal., v. 72, p. 295-310, 1991. 